\title{
Use of Material-Shape Factors in Mechanical Design
}

Filip Hrdlicka, Martin Kratochvil, Ivana Mazinova, Pavel Florian

Faculty of Mechanical Engineering, University of West Bohemia. Univerzitni 8, 30100 Plzen. Czech Republic. E-mail: ficeriov@kks.zcu.cz, kratochv@kks.zcu.cz, mazini@kks.zcu.cz,pflorian@kks.zcu.cz

During design process materials and their shapes selection is usually performed unsystematically, selection is mostly based on previous experience, although this is the most influencing part during mechanical design. This paper is devoted to the application of the material-shape factors and indexes to the standard bicycle frame. Paper to show the method with material-shape factors and indexes and how to use it for a simply and fast redesign for a different material and shapes. The whole method is descripted in detail for two examples and the results are verified by FEM analysis.

Keywords: shape factor; material index; optimization; mechanical design

\section{Introduction}

When we want to design or redesign new structure and find the best ratio between lightness and stiffness we can use two ways how to reach it. First way is topological optimization. This optimization can be used by some of computer designing program to calculate ideal shape of a construction. Second way is using the "trial and error" method, when after first design and calculation the structure is modified and calculated again. All this process is repeated so long, until the appropriate solution is found. But what if we want to make this designing part faster but do not have the possibility to use topological optimization? In this situation can be used material-shape factors as a simple and useful tool.

\section{Material-SHAPE factors}

\subsection{Material index}

Tab. 1 Design requirements [1]

\begin{tabular}{|l|l|l|}
\hline \multicolumn{2}{|l|}{ Design requirements } & Design requirements of cantilever beam \\
\hline Function & What does component do? & $\begin{array}{l}\text { A cantilever beam with loaded by force on free end, } \\
\text { circular cross-section }\end{array}$ \\
\hline Constrains & What conditions must be met? & Requires stiffness S and length L. \\
\hline Objective & What is to be optimized? & Minimize mass of the beam \\
\hline Free variable & Which parameters are free to change? & Radius r (half of diameter) \\
\hline
\end{tabular}

Objective equation of the beam loaded by a force is the mass equation of the beam

$$
\boldsymbol{m}=\boldsymbol{A L \rho}
$$

Where cross-section area $A$ depend on radius $r$

$$
A=\pi r^{2}
$$

In this case, the objective function contains free variable $r$, it must to be eliminated. Stiffness influence also missing, it can be used for substitution

$$
S=\frac{3 E I}{F L^{3}} \Rightarrow I=\frac{F L^{3} S}{3 E}
$$

The last step is to create relationship between crosssection area $A$ and cross-section moment of inertia $I$

$$
I=\frac{\pi}{4} \cdot r^{4}=\frac{\pi^{2} r^{4}}{4 \pi}=\frac{A^{2}}{4 \pi} \Rightarrow A=(4 \pi I)^{\frac{1}{2}}
$$

Then by substituting of two previous equations into objective function

$$
m=L \rho\left(4 \pi \cdot \frac{F L^{3} S}{3 E}\right)^{\frac{1}{2}}=\frac{\rho}{E^{\frac{1}{2}}}\left(4 \pi \cdot \frac{F L^{5} S}{3}\right)^{\frac{1}{2}}
$$

Material indices are a tool to assess suitability of a material for a specific application. Material index is mathematical formula, where input parameters are material properties and output is a value that describes the suitability of a material for a selected application. The higher value, the better material is for the selected application. This property of material indices makes it possible to compare the materials with each other and select the best material for the specific application. Integration of material indices with a material database creates a powerful tool for material selection (CES EduPack). To use material indices, it is necessary to define design requirements according to Table 1.

The principle of creation of the material indices is usually elimination of free variables in equation of objective function. Then it is necessary to group the material parameters. For example, design requirements of a beam loaded by a force on the free end are specified in Table 1.
Where $\frac{\rho}{E^{\frac{1}{2}}}$ formula describes the influence of material properties on beam stiffness. Material index value should be higher for better material so material index equation is in form

$$
M^{e}=\left(\frac{\rho}{E^{\frac{1}{2}}}\right)^{-1}=\frac{E^{\frac{1}{2}}}{\rho}
$$

According to the equation 6 can be created the material index for strength, where $\sigma_{f}$ is yield strength. [1]

$$
M^{f}=\frac{\sigma_{f}^{\frac{2}{3}}}{\rho}
$$

Another option is to create the material index using FEM. This approach is very effective in the case of a complex mathematical description of a physical phenomenon. However, advanced numerical analysis of FEM output is required to derive the material index. 


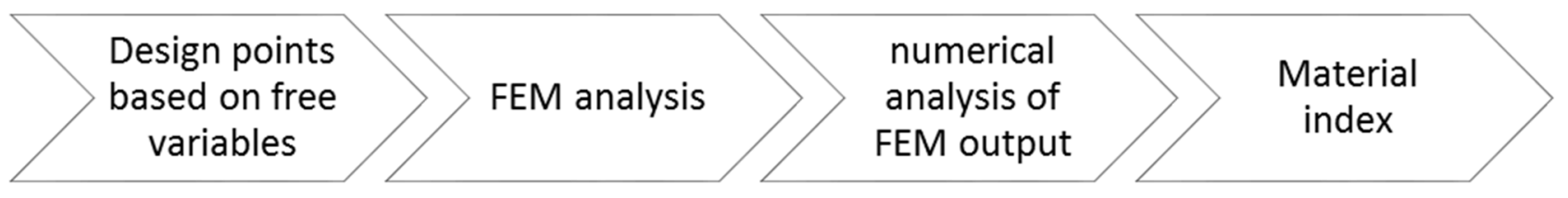

Fig. 1 Creating material index using FEM

Material index can be displayed in a material map to better explanation of the suitability of materials based on its properties. Figure 2 compare the suitability of the basic material against different material indices. In addition to material selection, material maps with material indices are excellent learning aids to understand the relationship between material properties and design requirements. [1]

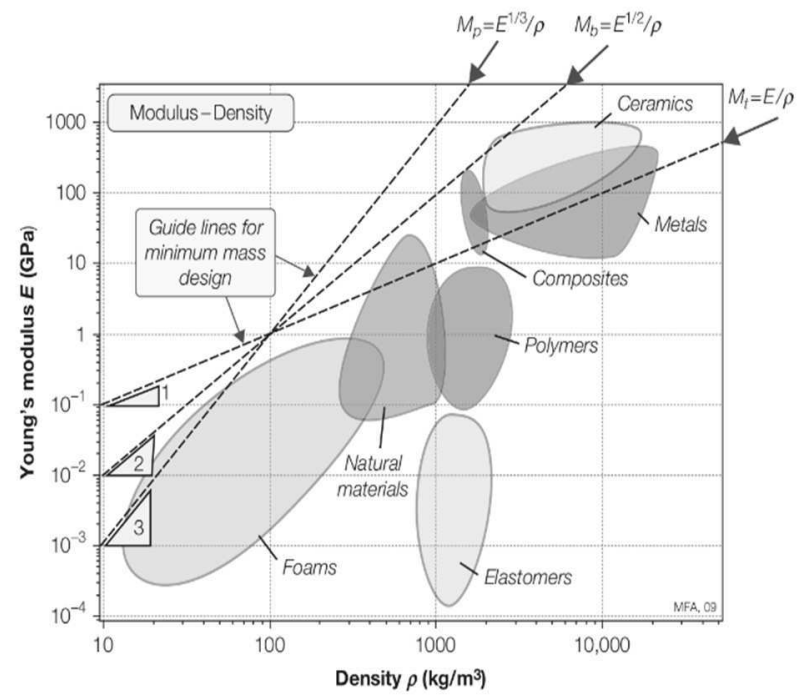

Fig. 2 Example of material map with a graphical representation of some materiel indices [1]

\subsection{Shape factor}

Shape factor is a tool for determining the suitability of a shape for specific application. It is developed for the case of uneven distribution of stresses in the crosssection. For this reason, shape factors are use for bending or torsion of a structure. It is further assumed that the shape factor is not dependent on the cross-section area. The principle of the function of the shape factors is to compare some shape to any cross section to square cross section. The shape factors can be created using analytical or numerical solution. It is important that there are two types of shape factors for any shape, elastic and plastic. Elastic shape factor is use for deformation, plastic shape factor is use for the evaluating of the stress influence or the evaluating of failure.

For the understanding, it is best to make a sample elastic shape factor derivation for a circular cross-section in the case of bending. [1]

Cross section area of square $A_{0}$ :

$$
A_{0}=a^{2}
$$

And its moment of inertia $I_{0}$

$$
I_{0}=\frac{a^{4}}{12}=\frac{A^{2}}{12}
$$

Cross-section area of circular shape $A$

$$
A=\pi a^{2}
$$

And its moment of inertia $I$

$$
I=\frac{\pi a^{4}}{4}=\frac{\pi^{2} a^{4}}{4 \pi}=\frac{A^{2}}{4 \pi}
$$

Elastic shape factor of circular cross-section

$$
\phi_{B}^{e}=\frac{I}{I_{0}}=\frac{A^{2}}{4 \pi} \cdot \frac{12}{A^{2}}=\frac{3}{\pi}
$$

According to the equation 12 can be created the shape factor for strength where $\mathrm{Z}$ is the bending cross section modulus.

$$
\phi_{B}^{f}=\frac{Z}{Z_{0}}=\frac{3}{2 \sqrt{\pi}}
$$

\subsection{Combination of material indices and shape factors}

Material indices or shape factors can be used separately, however, to assess the level of maturity of a design there is a possibility of their interconnection. The essence of connection is the transformation of material properties by shape factor. Evaluation of suitability is evaluated by comparison of material index values of transformed materials.

The material index combined with the elastic shape factor is created similarly to pure material index, but elimination of free variables must be based on selected shape factor. In this case, it is necessary to replace $I_{0}$ by the equation of its area and create the equation of moment of inertia based on cross section area. [1]

$$
\phi_{B}^{e}=\frac{I}{I_{0}}=I \cdot \frac{12}{A^{2}} \Rightarrow I=\frac{\phi_{B}^{e} A^{2}}{12}
$$

Following procedure is the same as the creation of the material index.

$$
\begin{aligned}
& S=\frac{3 E I}{F L^{3}}=\frac{3 E}{F L^{3}} \cdot \frac{\phi_{B}^{e} A^{2}}{12} \Rightarrow A=\left(\frac{4 F L^{3} S}{E \phi_{B}^{e}}\right)^{\frac{1}{2}} \\
& m=L \rho\left(\frac{4 F L^{3} S}{E \phi_{B}^{e}}\right)^{\frac{1}{2}}=\frac{\rho}{\left(E \phi_{B}^{e}\right)^{\frac{1}{2}}}\left(4 \pi \cdot F L^{5} S\right)^{\frac{1}{2}}
\end{aligned}
$$

At the end, the material-shape factor for bending consist of material parameters and shape factor.

$$
M_{B}{ }^{e}=\frac{\left(\phi_{B}^{e} E\right)^{\frac{1}{2}}}{\rho}
$$

According to the equation 17 can be created the material-shape factor for strength.

$$
M_{B}^{f}=\frac{\left(\phi_{B}^{f} \sigma_{f}\right)^{\frac{2}{3}}}{\rho}
$$




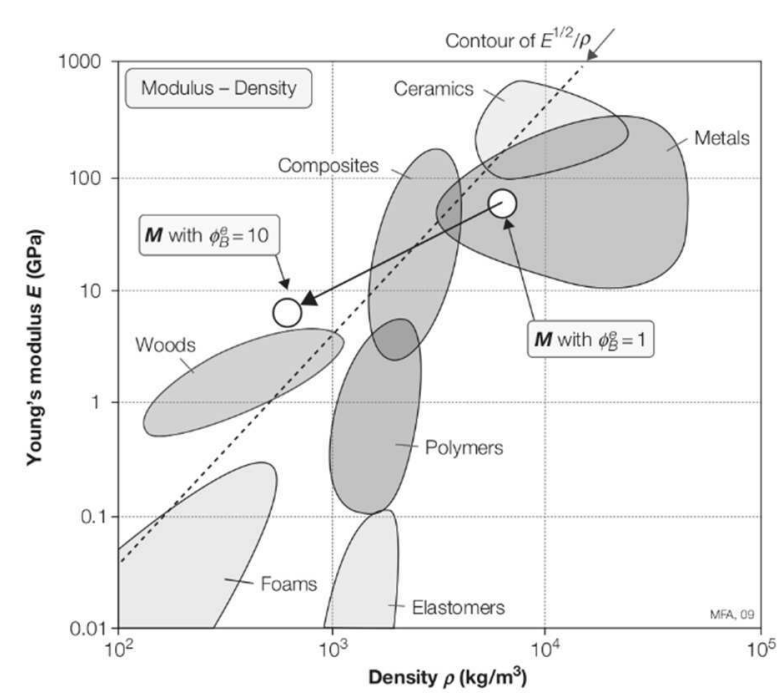

Fig. 3 Example of change of material index value based on shape factor [1]

\subsection{Material-Shape indexes}

Material-shape factors can show which combination is better in which category (stiffness, strength) but cannot show nothing more. For this case we create indexes which are based on the shape factors.

The first index is for mass. Lightness of a profile depends on volume and density. When we will compare two profiles of the different materials but the same length, then we need only cross-sections of the profiles and densities of both materials.

Equation of the index for light profile has form:

$$
i_{m}=\frac{m_{1}}{m_{2}}=\frac{A_{1} \rho_{1}}{A_{2} \rho_{2}} \geq 1
$$

Where result of the equation bigger than 1 means lighter profile than original one.

Similarly we can get an index for rigid profile in bending which is based on the equation 3 . This index depends on the ratio of Young's modulus and the second moment of area of both materials.

$$
i_{s}=\frac{s_{2}}{s_{1}}=\frac{E_{2} I_{2}}{E_{1} I_{1}} \geq 1
$$

Where result of the equation bigger than 1 means stiffer profile than original one.

Both equations we can combine into one and get combined index for stiff and light profile.

$$
i_{c}=\frac{i_{m}+i_{s}-2}{\sqrt{\left(i_{m}-1\right)^{2}+\left(i_{s}-1\right)^{2}}} \geq 1
$$

If the result is higher than 1 , it means that the new profile is lighter and stiffer than the original one. If the result is lower than -1 , it means that the new profile is heavier and flexible than the original one. If the result is between $(-1 ; 1)$, it means that the new profile is either lighter and flexible, or heavier and stiffer than the original one.

\section{Example of use}

With this method can be compared materials for every construction which is made from profiles like is construction of hang-glider, extension ladder or cabin for agriculture machine. [2][3] For our needs we use as an example aluminium alloy bicycle frame.

This frame is a common bicycle frame which has nowadays almost every bicycle. Advantages of this frame is low weigh, good stiffness and cheap price for profiles. But the cost of work, as welding and normalization, is much more expensive, then for steel. In the past, the steels do not have enough high yield strength to create stiff and light construction, but with the high strength steels (HSS) whole situation changed. [4] In this example we will try to design a steel bicycle frame with the same output dimensions, approximately stiffness and lightness as the aluminum frame.

The aluminium frame consist of 7 different profiles as shown in Figure 4. Profiles 1, 4 and 5 are depends on the inner diameter, as these profiles must be prepare for connecting standard parts of the bicycle as a saddle tube, handlebars and shaft hub. Profiles 6 and 7 are the same and profile no. 3 is profile of the elliptical shape.

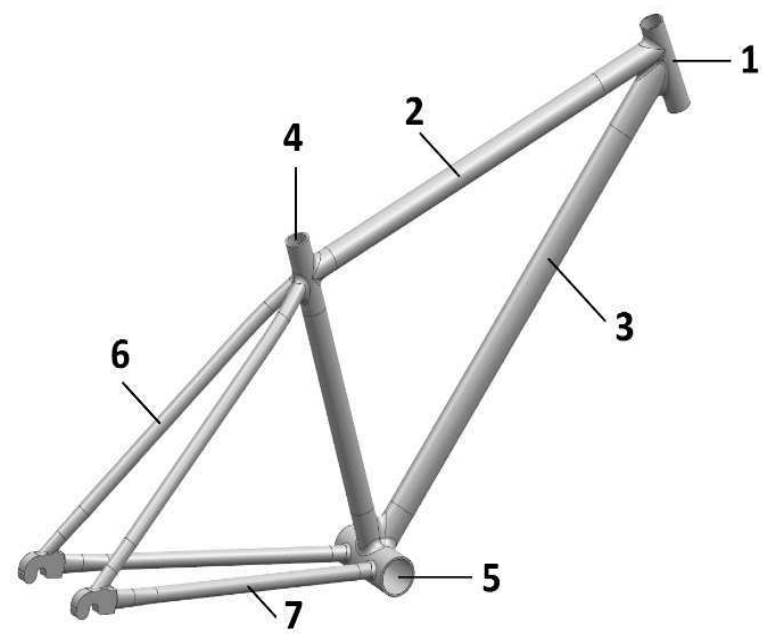

\begin{tabular}{|l|l|}
\hline Number of profile & Dimension $[\mathbf{m m}]$ \\
\hline 1 & $\emptyset 32 \times 2$ \\
\hline 2 & $\emptyset 32 \times 3$ \\
\hline 3 & $40 \times 30 \times 3$ \\
\hline 4 & $\emptyset 32 \times 3$ \\
\hline 5 & $\emptyset 52 \times 3$ \\
\hline 6 & $\emptyset 20 \times 2$ \\
\hline 7 & $\emptyset 20 \times 2$ \\
\hline
\end{tabular}

Fig. 4 Numbers of profiles 
The original material is the aluminium alloy AW 6061 T6 and as the comparative material will be used HSS Reynolds 531. Input values for calculation are density, Young's modulus and yield strength of both materials and dimension of the profiles of the original frame. Profiles are loaded by bending.

Tab. 1 Properties of materials

\begin{tabular}{|l|l|l|}
\hline Material & AW 6061 T6 & HSS \\
\hline Density [kg/m3] & 2700 & 7850 \\
\hline Young's module [GPa] & 70 & 210 \\
\hline Yield strength [MPa] & 250 & 880 \\
\hline $\boldsymbol{M}^{\boldsymbol{e}}$ & 97.99 & 58.75 \\
\hline $\boldsymbol{M}^{\boldsymbol{f}}$ & 14.69 & 11.69 \\
\hline $\boldsymbol{\phi}_{\boldsymbol{B} \text { max }}^{\boldsymbol{e}}[\mathbf{1 ]}$ & 38.486 & 35.530 \\
\hline $\boldsymbol{\phi}_{\boldsymbol{B} \max }^{\boldsymbol{f}}[\mathbf{1}]$ & 6.204 & 5.961 \\
\hline
\end{tabular}

According to the equation 6 and 7, it is clear that due to Ashby theory is for elasticity and strength better aluminium because it has higher values of material indices in both categories.

\subsection{Dsigning of profile 1}

The dimensions of the first profile are limited by the requirement of the inner diameter which must to be equal to $\mathrm{d}_{2}=28 \mathrm{~mm}$. For this condition we need to upgrade equation for $i_{m}$.

$$
A_{12} \text { max } \leq \frac{\rho_{1}}{\rho_{2}} \cdot A_{11}
$$

Where $A_{1}$ and $A_{2 \max }$ :

$$
\begin{gathered}
A_{11}=\frac{\pi}{4}\left(D_{11}{ }^{2}-d_{11}{ }^{2}\right) \\
A_{12 \text { max }}=\frac{\pi}{4}\left(D_{12}{ }^{2}-d_{12}{ }^{2}\right)
\end{gathered}
$$

Then:

$$
\begin{aligned}
& \frac{\pi}{4}\left(D_{12}{ }^{2}-d_{12}{ }^{2}\right) \leq \frac{\rho_{1}}{\rho_{2}} \cdot \frac{\pi}{4} \cdot 4\left(D_{11} t_{11}-t_{11}^{2}\right) \\
& D_{12 \max } \leq \sqrt{\frac{\rho_{1}}{\rho_{2}} \cdot 4\left(D_{11} t_{11}-t_{11}^{2}\right)-d_{12}^{2}}
\end{aligned}
$$

After values have been set we got result:

$$
D_{12} \leq 29.437 \mathrm{~mm}
$$

If we want this profile lighter than aluminium, then we need to choose an outside diameter smaller than $29.437 \mathrm{~mm}$. If we choose $\mathrm{D}_{12}=29 \mathrm{~mm}$, then $\mathrm{t}_{12}=0.5 \mathrm{~mm}$ and combine index will be:

$$
i_{c 1}=0.154
$$

That means that the HSS profile is lighter but flexible than the original one. If we calculate other indices, we can see how much it is.

$$
\begin{aligned}
i_{m 1} & =1.448=>44.8 \% \\
i_{S 1} & =0.640=>-36 \%
\end{aligned}
$$

The profile is almost $45 \%$ lighter but stiffness is lower about $36 \%$. We saved a lot of weight but we also lost a lot of stiffness. If $\mathrm{D}_{12}=30 \mathrm{~mm}$, than $\mathrm{t}_{12}=1 \mathrm{~mm}$ and combine index will be:

$$
\begin{gathered}
I_{c 1}=0.137 \\
i_{m 1}=0.712=>-28.8 \% \\
i_{S 1}=1.351=>35.1 \%
\end{gathered}
$$

Last try is select $\mathrm{D}_{2}$ as closer as possible to 29,437 $\mathrm{mm}$, then for $D_{12}=29.5 \mathrm{~mm}$ will be $t_{12}=0.75 \mathrm{~mm}$ and combine index will be:

$$
\begin{gathered}
i_{c 1}=-1.255 \\
i_{m 1}=0.957=>-4.3 \% \\
i_{S 1}=0.986=>-1.4 \%
\end{gathered}
$$

These result are pretty close to the original profile, but still a little bit worse. As we can see, in these small dimensions with this material we cannot reach better result in both categories.

The length of this profile is only $125 \mathrm{~mm}$ so selecting variation with bigger $i_{m 1}$ still does not save enough weight. Therefore, it will be better to use variation with larger $D_{12}$ which will increase stiffness of the first profile and also help to save weight to profiles 2 and 3 because these profiles are much longer. For this the best dimensions of first profile are $D_{12}=30 \mathrm{~mm}, t_{12}=1 \mathrm{~mm}$.

Before we can use this profile we need to check it if is not inclinable to buckling. For this we use Ashby's equation for tube:

$$
\phi_{B 1}^{f}=\frac{3}{\sqrt{2 \pi}} \cdot \sqrt{\frac{D_{12}}{2 t_{12}}} \leq \phi_{B \max }^{f}
$$

\subsection{Designing of profile 2}

The profile 2 is round tube in the original frame and does not have any requirements to shape. Only limit is width $30 \mathrm{~mm}$ because it must be connected to the profile 1.

The original dimensions of the profile 2 are $\mathrm{D}_{21}=32$ $\mathrm{mm}, \mathrm{t}_{21}=3 \mathrm{~mm}$. For round tube we know that $\mathrm{D}_{22}=30$ $\mathrm{mm}$ and only thickness of the tube is missing. According to equation 23 :

$$
\begin{gathered}
t_{22 \max } \leq \frac{D_{22}-\sqrt{D_{22}{ }^{2}-\frac{\rho_{1}}{\rho_{2}} \cdot 4\left(D_{21} t_{21}-t_{21}{ }^{2}\right)}}{2} \\
t_{22 \max } \leq 1.033 \mathrm{~mm}
\end{gathered}
$$

For $\mathrm{D}_{22}=30 \mathrm{~mm}$ and $\mathrm{t}_{22}=1 \mathrm{~mm}$ shape factors will be:

$$
\begin{gathered}
i_{c 2}=0.676 \\
i_{m 2}=1.032=>3.2 \% \\
i_{S 2}=0.991=>-0.9 \%
\end{gathered}
$$

This HSS profile is almost equal to aluminium and we cannot get higher values for one index without we lose in second one. But if we use the elliptical shape of the tube, we can get better results.

For this case, the width must be equal to $30 \mathrm{~mm}$ and thickness less than $1 \mathrm{~mm}$, so if $\mathrm{a}_{22}=30 \mathrm{~mm}$ and $\mathrm{t}_{22}=0.8$ $\mathrm{mm}$ then $\mathrm{b}_{22} \max$ will be:

$$
\begin{aligned}
& A_{22}=\frac{\pi}{2} \cdot t_{22}\left(a_{22}+b_{22}-2 t_{22}\right) \\
& b_{22} \max \leq \frac{8 \cdot \frac{\rho_{1}}{\rho_{2}} \cdot\left(D_{21} t_{21}-t_{21}{ }^{2}\right)}{\pi t_{22}}-a_{22}+2 t_{22} \\
& b_{22 \max } \leq 46.409 m m
\end{aligned}
$$

If $b_{22}=46 \mathrm{~mm}$, then:

$$
\begin{gathered}
i_{c 2 a}=1.038 \geq 1 \\
i_{c 2 b}=1.005 \geq 1 \\
i_{m 2}=1.005=>0.5 \%
\end{gathered}
$$




$$
\begin{gathered}
i_{S 2 a}=1.141=>14.1 \% \\
i_{S 2 b}=2.202=>120.2 \%
\end{gathered}
$$

$$
I_{22}=\frac{\pi}{64} \cdot b_{22}\left[a_{22}{ }^{3}-\left(a_{22}-2 t_{22}\right)^{3}\right]+2 t_{22}\left(a_{22}-2 t_{22}\right)^{3}
$$

$$
b_{22 \min } \geq \frac{\frac{E_{1}}{E_{2}}\left[D_{21}{ }^{4}-\left(D_{21}-2 t_{21}\right)^{4}\right]-2 t_{22}\left(a_{22}-2 t_{22}\right)^{3}}{a_{22}{ }^{3}-\left(a_{22}-2 t_{22}\right)^{3}}
$$

If $\mathrm{b}_{22}=40 \mathrm{~mm}$, then

$$
\begin{gathered}
i_{c 2 a}=1.156 \geq 1 \\
i_{c 2 b}=1.146 \geq 1 \\
i_{m 2}=1.094=>9.4 \% \\
i_{S 2 a}=1.016=>1.6 \% \\
i_{S 2 b}=1.582=>58.2 \%
\end{gathered}
$$

For checking profile for buckling we need to use Ashby's equation for ellipse shaped tube:

$$
\begin{gathered}
\phi_{B 2}^{f}=\frac{3}{\sqrt{2 \pi}} \cdot \sqrt{\frac{a_{22}}{t_{22}}} \cdot \frac{\left(1+\frac{3 b_{22}}{a_{22}}\right)}{\left(1+\frac{b_{22}}{a_{22}}\right)^{\frac{3}{2}}} \leq \phi_{B \max }^{f} \\
\phi_{B 2}^{f}=5.141 \leq 5.961
\end{gathered}
$$

As we can see, this profile is $9.4 \%$ lighter than the original one, stiffer in axis " $b$ " by $58.2 \%$ and in axis "a" is stiffness almost identical. Checkup for buckling is all right though we are close to the maximum limit of material-shape factor.
According to the equation 17 and 18, we can calculate values of the shape factor and material-shape factor for both materials with this round tube as is shown in Table 3.

Tab. 3 Material-shape values

\begin{tabular}{|l|l|l|}
\hline Material & AW 6061 T6 & HSS (REY. 531) \\
\hline $\boldsymbol{\phi}_{\boldsymbol{B}}^{\boldsymbol{e}}$ & 5.09 & 14.32 \\
\hline $\boldsymbol{\phi}_{\boldsymbol{B}}^{\boldsymbol{f}}$ & 2.76 & 4.63 \\
\hline $\boldsymbol{M}_{\boldsymbol{B}}{ }^{\boldsymbol{e}}$ & 221.14 & 236.72 \\
\hline $\boldsymbol{M}_{\boldsymbol{B}}{ }^{\boldsymbol{f}}$ & 28.94 & 34.84 \\
\hline
\end{tabular}

Although the material indices for elasticity and strength are better for material aluminium (Table 2), from the Table 3 it is clear that combination with the appropriate shape can be reach different result at the end. Comparing of material-shape factors clearly say, that for elasticity and strength is HSS better now.

\subsection{Results of the rest of profiles}

With the same process we can calculate, check and compare other profiles with the original ones. Results are listed in tables below.

Tab. 4 Results of HSS profiles

\begin{tabular}{|l|l|l|l|l|l|}
\hline Number of profile & Dimension $[\mathbf{m m}]$ & $\boldsymbol{i}_{\boldsymbol{S a}}[\mathbf{\%}]$ & $\boldsymbol{i}_{\boldsymbol{S} \boldsymbol{b}}[\mathbf{\%}]$ & $\boldsymbol{i}_{\boldsymbol{m}}[\mathbf{\%}]$ & Weight saving [g] \\
\hline $\mathbf{1}$ & $\emptyset 30 \times 1$ & 35.1 & 35.1 & -28.8 & -25.78 \\
\hline $\mathbf{2}$ & $30 \times 40 \times 0.8$ & 1.6 & 58.2 & 9.4 & 36.42 \\
\hline $\mathbf{3}$ & $30 \times 50 \times 0.8$ & 18.7 & 0.1 & 5.3 & 18.82 \\
\hline $\mathbf{4}$ & $\emptyset 30 \times 1$ & -0.9 & -0.9 & 3.2 & 10.93 \\
\hline $\mathbf{5}$ & $\varnothing 48 \times 1$ & -12 & -12 & 7.6 & 10.54 \\
\hline $\mathbf{6}$ & $20 \times 24 \times 0.5$ & 8.7 & 43.9 & 15.2 & $2 \times 19.32$ \\
\hline $\mathbf{7}$ & $20 \times 24 \times 0.5$ & 8.7 & 43.9 & 15.2 & $2 \times 17.31$ \\
\hline
\end{tabular}

Tab. 5 Comparison of shape factors for $A W$ and HSS

\begin{tabular}{|l|l|l|l|l|}
\hline Number of profile & $\boldsymbol{\phi}_{\boldsymbol{B}}^{\boldsymbol{e}} \mathbf{A W}[\mathbf{1}]$ & $\boldsymbol{\phi}_{\boldsymbol{B}}^{\boldsymbol{e}} \mathbf{H S S}[\mathbf{1}]$ & $\boldsymbol{\phi}_{\boldsymbol{B}}^{\boldsymbol{f}} \mathbf{A W}[\mathbf{1}]$ & $\boldsymbol{\phi}_{\boldsymbol{B}}^{\boldsymbol{f}}$ HSS [1] \\
\hline $\mathbf{1}$ & 7.639 & 14.324 & 3.385 & 4.635 \\
\hline $\mathbf{2}$ & 5.093 & 16.443 & 2.764 & 5.141 \\
\hline $\mathbf{3}$ & 4,385 & 15.107 & 2.655 & 5.049 \\
\hline $\mathbf{4}$ & 5.093 & 14.324 & 2.764 & 4.635 \\
\hline $\mathbf{6}$ & 8.276 & 22.918 & 3.523 & 5.863 \\
\hline $\mathbf{7}$ & 4.775 & 18.152 & 2.676 & 5.335 \\
\hline
\end{tabular}

Tab. 6 Comparison of material-shape factors for $A W$ and HSS

\begin{tabular}{|l|l|l|l|l|}
\hline Number of profile & $\boldsymbol{M}_{\boldsymbol{B}}{ }^{\boldsymbol{e}} \mathbf{A W}[\mathbf{1}]$ & $\boldsymbol{M}_{\boldsymbol{B}}{ }^{\boldsymbol{}} \mathbf{H S S}[\mathbf{1}]$ & $\boldsymbol{M}_{\boldsymbol{B}}{ }^{\boldsymbol{}} \mathbf{A W}[\mathbf{1 ]}$ & $\boldsymbol{M}_{\boldsymbol{B}}{ }^{\boldsymbol{f}} \mathbf{H S S ~ [ 1 ] ~}$ \\
\hline $\mathbf{1}$ & 270.84 & 220.94 & 33.14 & 32.52 \\
\hline $\mathbf{2}$ & 221.14 & 236.72 & 28.95 & 34.84 \\
\hline $\mathbf{3}$ & 205.19 & 226.90 & 28.18 & 24.43 \\
\hline $\mathbf{4}$ & 221.14 & 220.94 & 28.95 & 32.52 \\
\hline $\mathbf{5}$ & 281.90 & 279.47 & 34.03 & 38.04 \\
\hline $\mathbf{6}$ & 214.12 & 248.71 & 28.33 & 35.72 \\
\hline $\mathbf{7}$ & 214.12 & 248.71 & 28.33 & 35.72 \\
\hline
\end{tabular}


In Table 4 we can see that all HSS profiles have higher or same stiffness as the original ones expect profile No. 5 and total weight saving is equal to $124.184 \mathrm{~g}$. If we compare values in Table 5 with Table 2 we find out that profile No. 5 is also very close to the maximum limit of material-shape factor for buckling. This could be dangerous, but because the shaft hub for pedals will be placed inside, the whole profile will be reinforced. By comparison of these two tabs we can also see that the aluminium alloy has a higher potential of use than the HSS, because factors of the HSS for buckling are very close to the limit.

From Table 6 is obvious that almost all HSS profiles have better stiffness and strength than aluminium profiles. This means that results of FEM analysis should say that HSS frame will be stiffer and stronger than the aluminium frame.

\subsection{Computer model and FEM}

With dimensions of profiles from the Table 4 was created a $3 \mathrm{D}$ computer model of bicycle frame and it was

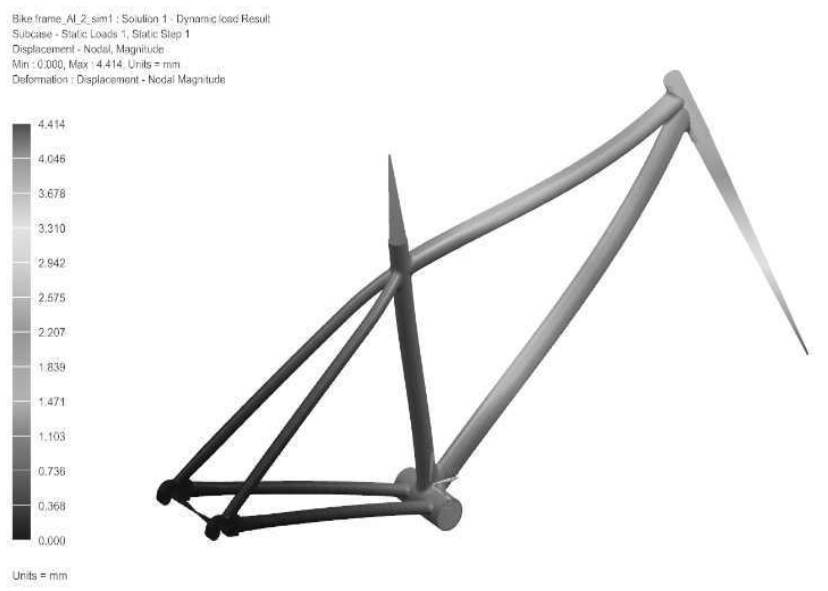

compared to the original one. The premise says that HSS frame should be slightly stiffer and lighter than the original one.

Total weight of aluminium frame is:

$$
m_{A W}=2.196 \mathrm{~kg}
$$

Total weight of HSS frame is:

$$
m_{H S S}=2.322 \mathrm{~kg}
$$

As we see, the HSS frame is slightly heavier than the aluminium, how is it possible? There are two reasons. First, the frame is composed of profiles and holders of the rear wheel. These holders are made of solid material of the same volume, so the HSS holders are heavier than the aluminium ones. Second, the frame is welded so that it is necessarily count with extra weight of the welds, where the HSS welds have a higher weight again.

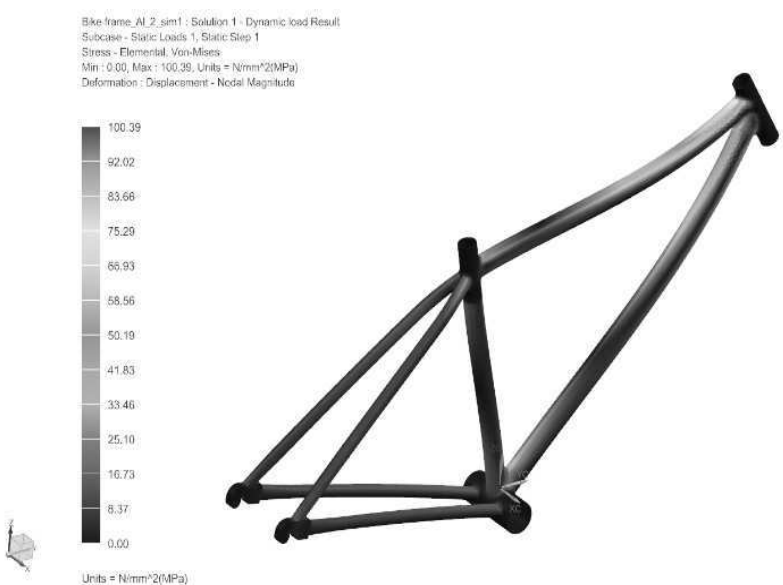

Fig. 5 Deformation and stress of aluminum frame
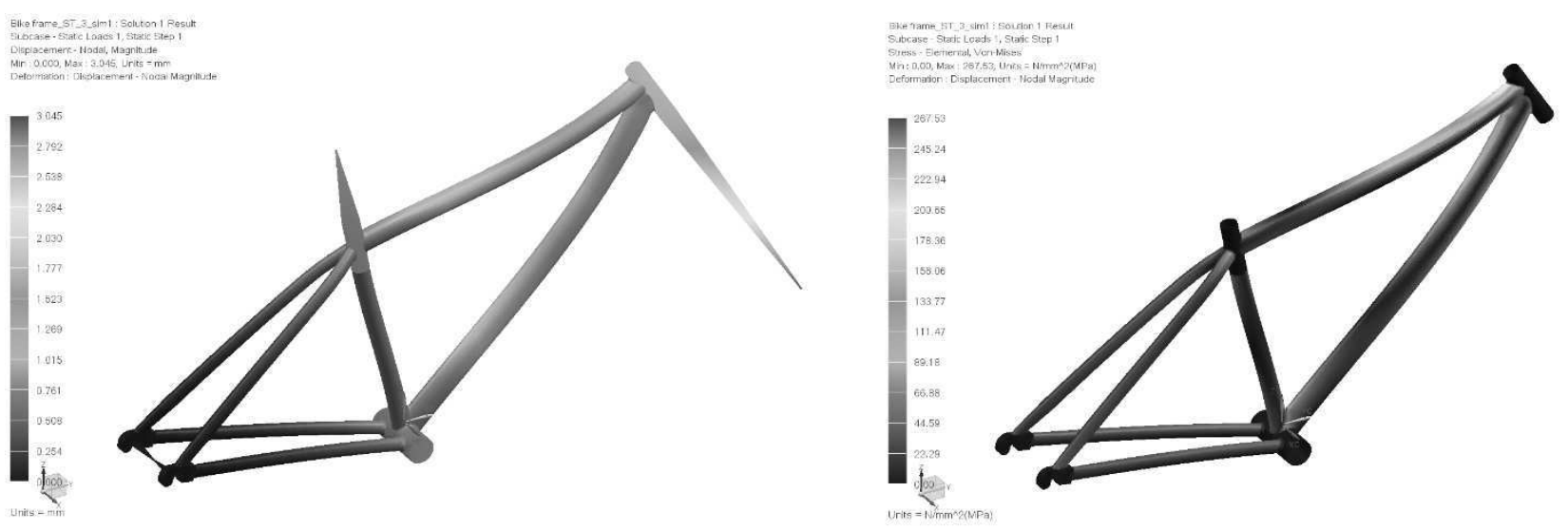

Fig. 6 Deformation and stress of HSS frame

Frames were loaded by two forces. First equal to $2000 \mathrm{~N}$ was placed in the middle of profile No. 5 . The second one was placed on the top of profile No. 1. equal to $1000 \mathrm{~N}$. This distribution of forces corresponds to the dynamic load of the cyclist during impact after jump.

Deformation of aluminium frame is $4,41 \mathrm{~mm}$ and maximum stress is $100,39 \mathrm{MPa}$. Deformation of HSS frame is 3,05 $\mathrm{mm}$ and maximum stress is $267,53 \mathrm{MPa}$.

If we compare the maximum values of stress both frames with the yield modulus of both materials, we reach the safety factor of the structure.

For aluminium frame:

$$
k_{A W}=\frac{\sigma_{f}}{\sigma_{A W}}=\frac{250}{100.39}=2.49
$$


For HSS frame:

$$
k_{S T}=\frac{\sigma_{f}}{\sigma_{S T}}=\frac{880}{267.53}=3.29
$$

The safety factor of the HSS frame is $1,3 \mathrm{x}$ higher than aluminium one. This means that HSS is stronger than aluminium alloy and we can pick up steel with lower yield modulus. If we would want the same value of safety, we need to find a steel with a yield modulus close to 666 $\mathrm{MPa}$.

By comparing the results from Table 6 with the results from FEM analysis it is clear that HSS frame is really stronger and stiffer than the aluminium and the prediction made by material-shape factors was correct.

\section{Conclusion}

The goal of this paper was to show the possibilities of using material-shape factors for the design of profile structures. With a combination of Microsoft Excel we can get quick, easy and powerful tool for structure optimization who can be used by everyone.

\section{Acknowledgments}

This study was supported by the internal project of University of West Bohemia SGS-2019-001.

\section{References}

[1] ASHBY, M. F. (2011). Material Selection in Mechanical Design, pp. 97 - 124, 243 - 277. Butterworth-Heinemann, Oxford. ISBN 978-185617-663-7

[2] KUBASEK, J., VOJTECH, D., DVORSKY, D. (2017). Structure and Mechanical Properties of Aluminium Alloy Sampled from a Firefighter Ladder. In: Manufacturing Technology, Vol. 17, No. 6, pp. 876-881.

[3] LIU, S., YANG, X. (2016). The Parametric Design of the Frame of Agricultural Machinery Cab based on Analysis of Er-gonomics Data, In: Manufacturing Technology, Vol. 16, No. 3, pp. 543551.

[4] BUBLIKOVÁ, D., JIRKOVA, H., BEHULOVÁ, M., KRAJCOVIC, J. (2019) Determination of Transformation Temperatures of Advanced HighStrength Steels and Their Use in Designing $Q \& P$ Process Routes, In: Manufacturing Technology, Vol. 19, No. 1, pp. 18-22. 